\title{
Rheumatology and the Kidney, 2nd Edition
}

Dwomoa Adu, Paul Emery, Michael Madaio. Oxford: Oxford University Press, April 2012, 466 pages, \$78 US

This book represents a joint effort by rheumatologists and nephrologists to describe many of the diseases treated by both subspecialties. Although many chapters are devoted to lupus nephritis and vasculitis [especially antineutrophil cytoplasmic antibody (ANCA)-associated vasculitis], the book covers a multitude of topics from nephrotoxicity of drugs used in rheumatology including analgesic nephropathy and nonsteroidal antiinflammatory drug toxicity to gout, amyloidosis, and hepatitis C virus-associated mixed cryoglobulinemia. Some topics are likely more relevant to only 1 specialty (e.g., Chapter 16 on Takayasu arteritis), but overall the topic selection is thorough. The text is generally well written but could benefit from color histology pictures, and in some chapters additional tables or figures could be added to better illustrate the main concepts. Not everyone is likely to read it cover to cover - it is probably better used as a reference book. The chapters on systemic lupus erythematosus and those on ANCA-associated vasculitis have considerable overlap that could be cut down in later editions to improve the flow of the text. Additionally, in the vasculitis chapters there is inconsistency in the nomenclature for the ANCA-associated vasculitides, with some chapters referring to the old nomenclature (e.g., Wegener's granulomatosis) and some to the newer (e.g., granulomatosis with polyangiitis).

The latter point highlights a problem with textbooks in rheumatology - our understanding of the immunology of these diseases, treatments, and even nomenclature can change rapidly, making textbooks quickly out of date. Unfortunately it does not appear that there is an online edition of this text, which would be amenable to such updates. Perhaps this could be a consideration in future editions. Despite these minor limitations, overall the breadth and depth of the text make the book excellent for trainees to study for rheumatology/nephrology examinations and as an office reference for practicing rheumatologists, nephrologists, and general internists.

CLAIRE E.H. BARBER, MD, FRCPC, Department of Medicine, Division of Rheumatology, University of Calgary, HRIC Room 3AA20, 3280

Hospital Drive NW, Calgary, Alberta T2N 4N6, Canada.

E-mail: cehbarbe@ucalgary.ca

J Rheumatol 2012;39:11; doi:10.3899/jrheum.121076 